Reprinted from The Journal of Chemical Physics, Vol. 20, No. 7, 1175-1176, July, 1952 Printed in U. S. A.

\title{
On the Validity of Anomalous Population Temperatures in Flames*
}

\author{
S. S. Penner \\ Guggenheim Jet Propulsion Center, California Institute of Technology,
Pasadena, California \\ (Received May 1, 1952)
}

$I^{\mathrm{T}}$ $T$ should be obvious to anyone reading our recent papers objectively that our "principal thesis" was (a) to examine the origin of the basic relations involved in the customary procedure for the determination of population temperatures in flames, with emphasis on the effect of spectral line shape on the final equations, ${ }^{1}$ and (b) to present experimental data on low pressure combustion flames ${ }^{2}$ which support the earlier experimental findings of Gaydon and Wolfhard. ${ }^{3}$ We noted that the usual equations hold only if $\left(P_{\nu}\right)_{\max } X \ll 1$, and pointed out some time ago $0^{4}$ that this condition is equivalent to the absence of self-absorption. Furthermore, contrary to Shuler's interpretation of our remarks, ${ }^{5}$ use of the best available intensity data, namely, the data obtained by Oldenberg and Rieke, ${ }^{6}$ led us to the conclusion that the condition $\left(P_{\nu}\right)_{\max } X \ll 1$ is apparently not satisfied in representative low pressure combustion flames. We therefore arrived at the conclusion that anomalous population temperatures reported for low pressure combustion flames ${ }^{3}$ may be the result of misinterpretation of experimental data.

Since we have done no experimental work on flames burning at atmospheric pressure, no reference was made to the attempts to correct for self-absorption in flames of this type. However, since Shuler ${ }^{5}$ has furnished us with a résumé of what he considers to be important with regard to the study of flames burning at atmospheric pressure, it may be desirable if we indicate our position with regard to this work. First of all, we wish to call attention to the many important contributions made by Dieke and his collaborators to the development of valid techniques for the determination of population temperatures. ${ }^{7}$ This work appears to have been done competently and with care. To the best of our knowledge, Dieke has not emphasized the occurrence of "anomalous" temperatures. We have not attempted investigations of flames burning at atmospheric pressure (1) because we were fortunate in having at our disposal the very excellent low pressure flame apparatus constructed by Gilbert, ${ }^{2}$ and (2) because we did not feel in a position to carry out an unambiguous analysis of data obtained by examination of very narrow reaction zones, particularly if the field of view contains strong temperature gradients.

A straightforward quantitative study has been carried out recently ${ }^{8}$ on the effect of self-absorption in an isothermal system

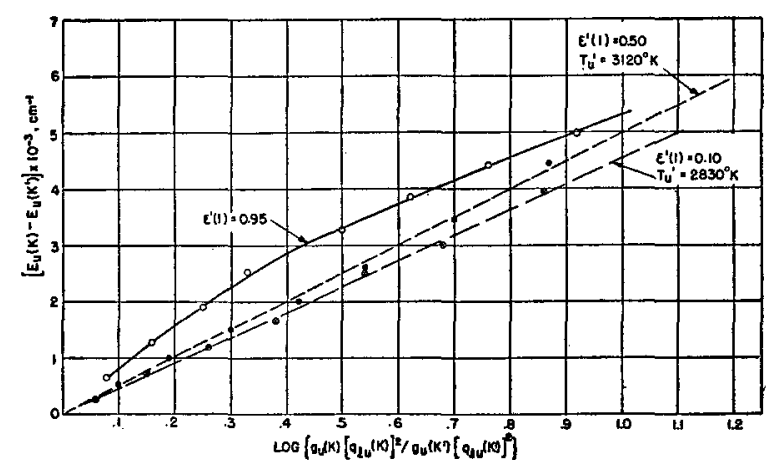

Fig. 1. Plot of $\left[E_{u}(K)-E_{u}\left(K^{\prime}\right)\right] v s \log \left\{g_{u}(K)\left[q_{l u}(K)\right]^{2 / g_{u}}\left(K^{\prime}\right)\left[q_{l u}\left(K^{\prime}\right)\right]^{2}\right\}$ for lines with equal total intensities at $3000^{\circ} \mathrm{K}$ as a function of $\epsilon^{\prime}(1)$ for the for lines with equal total intensities at $3000^{\circ} \mathrm{K}$ as a
$P_{1}$-branch, $(0,0)$-band, $2 \Sigma \rightarrow^{2} \Pi$ transitions of $\mathrm{OH}$. at $3000^{\circ} \mathrm{K}$ on the observable experimental data by using the quantity

$$
\epsilon^{\prime}(1)=1-\exp \left(-P_{\max } X\right)
$$

for the first line of the $P_{1}$-branch, $(0,0)$-band, ${ }^{2} \Sigma \rightarrow^{2} \Pi$ transitions of $\mathrm{OH}$, as a variable parameter. These detailed calculations lead to the conclusions enumerated below.

(1) Apparent population temperatures between 3000 and $19,000^{\circ} \mathrm{K}$ are obtained in conventional plots from the lines with $9 \leqslant K \leqslant 18$ as $\epsilon^{\prime}(1)$ is varied from 0.1 to 0.99 .

(2) Self-absorption must occur in absorption experiments for an isothermal system whenever it occurs in emission experiments since the spectral emissivities and absorptivities are identical. Nevertheless, it appears to be possible to correlate the results of absorption experiments by conventional plots even under conditions in which self-absorption is not negligible. However, the temperatures calculated ${ }^{9}$ from the slopes are not reliable. ${ }^{8}$

(3) As is implied by the work of Cowan and Dieke, ${ }^{7}$ the isointensity method of Shuler ${ }^{10}$ is not always a valid procedure, since the effects of self-absorption do not necessarily cancel in first order. This last statement is amplified by the curves shown in Fig. 1, which were constructed according to Shuler's method. ${ }^{10}$ Reference to Fig. 1 shows that the temperatures calculated from the slopes differ slightly from the known temperature of $3000^{\circ} \mathrm{K}$ for small values of $\epsilon^{\prime}(1)$ and that the curves become nonlinear for large values of $\epsilon^{\prime}(1)$. The absolute intensity estimates obtained by Oldenberg and Rieke ${ }^{\epsilon}$ are consistent with $0.3 \leqslant \epsilon^{\prime}(1) \leqslant 0.99$ for representative flames at $3000^{\circ} \mathrm{K}$.

On the basis of our present knowledge of his work and the results of our calculations, we do not feel that we can accept Shuler's "anomalous" vibrational temperatures ${ }^{11}$ as valid experimental evidence without (1) convincing proof that all necessary corrections for temperature gradients in the field of view have been applied, preferably by use of the methods of Cowan and Dieke, ${ }^{7}$ and (2) a quantitative demonstration, using the absolute intensity estimates of Oldenberg and Rieke, ${ }^{6}$ that the effects of self-absorption do not produce significant distortion of the experimental data.

* Supported by the ONR under Contract Nonr-220(03), NR 015210. 1 S. S. Penner, J. Chem. Phys, 20, 507 (1952). It should be noted that, in general, peak intensity ratios are obtained if the instrumental slit width is small compared to the line width, whereas total intensity ratios are
measured when the instrumental slit width is large compared to the line width.

width.

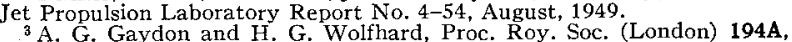

169 (1948), and later publications. 015210 , November 1951; Technical Report No. 4, February, 1952.

5 K. E. Shuler, J. Chem. Phys. (to be published).

$6 \mathrm{O}$. Oldenberg and F. F. Rieke, J. Chem. Phys. 6, 439 (1938); R. J. Dwyer and $O$. Oldenberg, $j$. Chem. Phys. 12, 351 (1944). The numerical values of maximum absorption coefficients listed in Table II of reference 1 must be used in conjunction with optical densities $X$ which represent the population of molecules in the lower energy level involved in a given energy

${ }_{7}$ R. D. Cowan and G. H. Dieke, Revs. Modern Phys. 20, 418 (1948); G. H. Dieke and H. M. Crosswhite, Bumblebee Report No. 87, November, 1948.

8 S. S. Penner. Technical Report No. 5, April, 1952 (to be published).

9 H. P. Broida, J. Chem. Phys. 19, 1385 (1951).

$11 \mathrm{H}$. P. Broida and K. E. Shuler, J. Chem. Phys. 20, 168 (1952). 
FIG. 4

PLOT OF $\left[E_{u}(K)-E_{u}\left(K^{\prime}\right)\right]$ vs. LOG $\left\{g_{u}(K)\left[q_{\ell u}(K)\right]^{2} / g_{u}\left(K^{\prime}\right)\left[q_{\ell u}\left(K^{\prime}\right)\right]^{2}\right\}$ FOR LINES

WITH EQUAL TOTAL INTENSITIES AT $3000 \mathrm{~K}$ AS A FUNCTION OF $\varepsilon^{\prime}(1)$ FOR THE

$P_{1}$ - BRANCH, $(0,0)-B A N D,{ }^{2} \Sigma \rightarrow^{2}$ II TRANSITIONS OF OH.

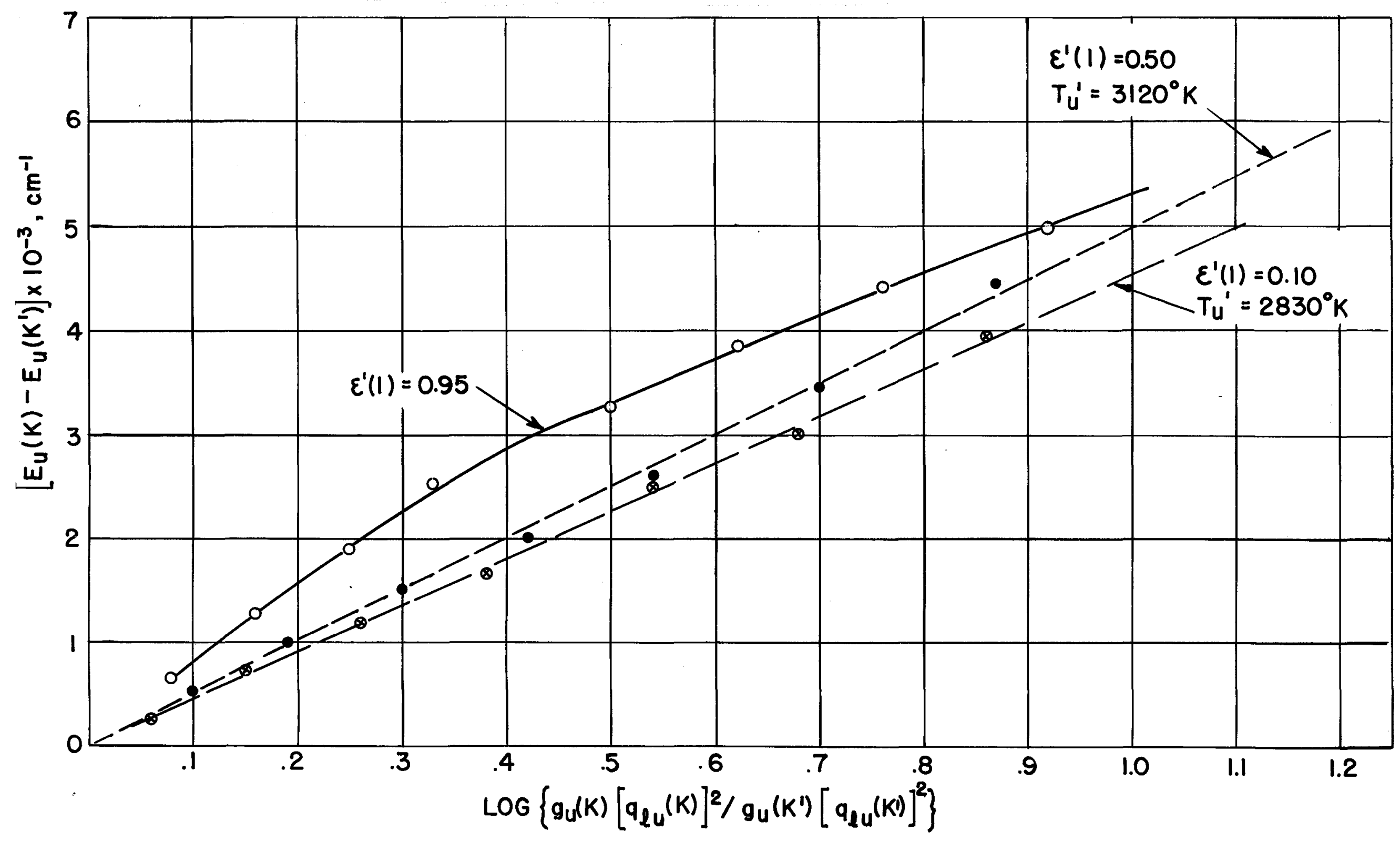

